\title{
Low-dose dexamethasone affects osteoblast viability by inducing autophagy via intracellular ROS
}

\author{
SHAOKUN ZHANG ${ }^{1}$, YONGYI LIU ${ }^{1}$ and QINGWEI LIANG ${ }^{2}$ \\ Departments of ${ }^{1}$ Orthopedics and ${ }^{2}$ Sports Medicine, The First Hospital of \\ China Medical University, Shenyang, Liaoning 110000, P.R. China
}

Received August 10,2017; Accepted December 18, 2017

DOI: $10.3892 / \mathrm{mmr} .2018 .8461$

\begin{abstract}
Glucocorticoids (GCs) are closely associated with the progression of GC-induced osteoporosis (GIOP) by inhibiting osteoblast viability. However, endogenous GCs are important for bone development. In addition, previous studies have demonstrated that GCs could induce autophagy, a cytoprotective process that is protective against various stressors. In the present study, the aim is to explore whether osteoblasts exhibited dose-dependent viability in the presence of GCs due to autophagy. hFOB 1.19 osteoblasts were treated with various doses of dexamethasone (DEX; $10^{-8}-10^{-4} \mathrm{M}$ ) for $0,24,48$ and $72 \mathrm{~h}$. The results revealed a biphasic effect of DEX on the viability of hFOB 1.19 cells; a high dose of DEX $\left(\geq 10^{-6} \mathrm{M}\right)$ accelerated cell apoptosis, while a low dose of DEX $\left(10^{-8} \mathrm{M}\right)$ increased cell viability. Furthermore, significantly increased autophagy was observed in the low dose DEX treatment group, as indicated by the expression of the autophagy-associated proteins beclin 1 and microtubule-associated protein light chain 3 , and the detection of autophagosomes. Another finding was that DEX upregulated intracellular reactive oxygen species (ROS), which was decreased by the autophagy agonist rapamycin. The increase in autophagy and cell viability associated with low-dose DEX $\left(10^{-8} \mathrm{M}\right)$ was suppressed by the ROS scavenger catalase and the autophagy inhibitor 3-methyladenine. In conclusion, the results revealed that GCs affected osteoblast viability in a dose-dependent manner. A low dose of GCs increased osteoblast viability by inducing autophagy via intracellular ROS. The results indicate that autophagy may be a novel mechanism by which osteoblasts survive GC exposure and provide a potential therapeutic target for treating GIOP.
\end{abstract}

Correspondence to: Professor Qingwei Liang, Department of Sports Medicine, The First Hospital of China Medical University, 155 North Nanjing Street, Shenyang, Liaoning 110000, P.R. China E-mail: lqwcmu@hotmail.com

Key words: dexamethasone, autophagy, osteoblasts, viability, reactive oxygen species

\section{Introduction}

Due to the outstanding performance with respect to immune response and anti-inflammatory effect, glucocorticoids (GCs) are routinely prescribed to treat autoimmune and noninfectious inflammatory diseases. However, the long-term and excessive administration of GCs can lead to many serious complications, such as osteoporosis. GC-induced osteoporosis (GIOP), which is considered to be the most common form of secondary osteoporosis $(1,2)$, increases the risk of fracture in patients treated with GCs. Clinical studies have indicated that GCs dysregulated bone metabolism and led to a rapid reduction in trabecular bone mass within the first year of treatment $(3,4)$. However, this reduction in trabecular bone mass cannot entirely explain the observed increase in fracture risk because the bone mineral density (BMD) value of patients treated with GCs is higher than those of women with postmenopausal osteoporosis (4). Therefore, it is necessary to more comprehensively elucidate the pathology of GC-induced bone loss. A better understanding of this process will help clinicians effectively prevent and treat GIOP.

Osteoblasts, which are derived from mesenchymal progenitor cells, are mainly responsible for bone formation and development (5). Prior studies have suggested that the administration of GCs induced the apoptosis of osteoblasts and osteocytes, which was regarded as the main cause of GIOP (6). Nevertheless, it is well known that GCs are indispensable for normal bone metabolism and development under physiological condition (7). A recent study demonstrated that different dose of GCs led to different osteocyte fates (8). In addition, Shi et al (9) concluded that GCs had dose-related effect on osteoclast formation and function. Based on these studies, GC dose is a crucial factor affecting the function and viability of cells. Unfortunately, few studies have addressed the relationship between osteoblast viability and GC dose, particularly for low-GC dose.

Autophagy is a highly conserved intracellular cleaning process in eukaryotic cells that is responsible for removing damaged organelles and counteracting harmful stimuli $(10,11)$. Generally, physiological autophagy exists in all cells to maintain normal cellular metabolism and viability (12). Once cells encounter stress stimuli, such as hypoxia (13), oxidative stress (14), or ER stress (15), autophagy is induced to prevent cells from undergoing apoptosis by eliminating stress 
inducers. Autophagy reportedly plays a protective role in maintaining osteoblast viability by allowing cells to survive various stresses $(16,17)$.

Microtubule-associated protein light chain 3 (LC3) and beclin 1 are important biological signal to identify autophagy (18). In the process of autophagy, cytoplasmic proteins, organelles and so on, are engulfed by autophagosomes. LC3II is simultaneously formed by conjugating LC3I with phosphatidylethanolamine, which is fixed on the membrane of autophagosome. After the formation of autolysosome consisting of lysosome and autophagosome, the cytoplasm in autophagosome is degraded. Meanwhile, LC3II is also degraded in the autolysosome. Therefore, the activity of autophagy could be characterized by detecting activity of LC3II $(18,19)$. Beclin 1 is also an essential protein for the formation of autophagosome. It can guide other autophagy-related proteins to move into vacuoles, and thus regulates the formation of autophagosome (20).

It is well-known that GCs can interrupt mitochondrial function and thereby increase reactive oxygen species (ROS) in many cells $(21,22)$. Generally, ROS is produced by mitochondria during cellular metabolism. However, ROS level is significantly increased when cells are exposed to noxious stimuli, such as cytotoxic drugs. At normal level, ROS plays a crucial role in the energy cycling of cells; however, excessive ROS induces cell apoptosis, even death, which are closely associated with many diseases $(23,24)$. Certain studies have indicated that reduced bone formation and decreased BMD were induced by increased oxidative stress in aged humans and mice $(25,26)$. Moreover, a recent study demonstrated that the GC-induced upregulation of ROS could lead to osteoporosis by inducing osteoblast apoptosis (2). However, studies have claimed that ROS, as a source of oxidative stress, also contributed to the initiation of autophagy to prevent osteoblasts from undergoing apoptosis (17). We hypothesize that the biphasic effect of ROS on osteoblast viability may be attributable to the difference in ROS level, which is associated with GC dose. In other words, GC dose determine osteoblast fate by modulating intracellular ROS levels. However, this hypothesis must be verified by additional research.

The primary aim of this study is to investigate the effect of GC dose on the viability of osteoblasts, assess whether autophagy is involved in this process, and examine the relationship between ROS and autophagy.

\section{Materials and methods}

Reagents and antibodies. Anti-LC3 and anti-beclin 1 antibodies were purchased from Cell Signaling Technology, Inc. (Danvers, MA, USA). Rapamycin (Rap, autophagy agonists), 3-methyladenine (3-MA, autophagy inhibitor) were purchased from Selleck Chemicals (Houston, TX, USA). Dexamethasone (DEX) was purchased from Sigma-Aldrich; Merck KGaA (St. Louis, MO, USA). The ROS scavenger catalase was purchased from Beyotime Institute of Biotechnology (Nantong, China).

Cell culture and treatment. The human fetal osteoblastic cell line hFOB 1.19 cells were kindly provided by Dr. M. Subramaniam. The cells were cultured in a 1:1 mixture of DMEM/F-12 supplemented with $10 \%$ fetal bovine serum and $0.3 \mathrm{~g} / 1 \mathrm{G} 418$ under $5 \% \mathrm{CO}_{2}$ humidified atmosphere at $33.5^{\circ} \mathrm{C}$. After the cells reached a steady-state of exponential growth in the media, they were exposed to variable concentrations of DEX for specific time. Rap (3 $\mu \mathrm{M})$, 3-MA (5 mM), and catalase $(500 \mathrm{U} / \mathrm{ml})$ were added to cultures, respectively where indicated.

Cell viability assay. The hFOB 1.19 cells were seeded into 96 -well plates $\left(4 \times 10^{3}\right.$ cells/well). When $60 \%$ confluence was achieved, cells were treated with indicated treatments. Cell viability was determined by 3-[4,5-dimethylthiazol-2-y]-2,5-diphenyltetrazolium bromide (MTT; Keygen, Nanjing, China) assay following the manufacturer's instructions.

Cell apoptosis analysis. After treated with indicated treatment for $24 \mathrm{~h}$, cells were collected and stained with the Annexin V-FITC Apoptosis Detection kit (Keygen) according to manufacturer's instructions, and the apoptosis was analyzed by the flow cytometer equipped with Modfit LT 3.0 (BD Biosciences, Franklin Lakes, NJ, USA). Different subpopulation was distinguished using the following criteria: Q3, viable cells (TITC-/PI'); Q4, early apoptotic cells $\left(\mathrm{FITC}^{+} / \mathrm{PI}^{-}\right)$; Q2, late apoptotic cells $\left(\mathrm{FITC}^{+} / \mathrm{PI}^{+}\right)$; Q1, necrotic cells $\left(\mathrm{FITC}^{-} / \mathrm{PI}^{+}\right)$. The apoptotic rate was determined as the percentage of $\mathrm{Q} 2+\mathrm{Q} 4$.

Intracellular ROS measurement. The ROS level of osteoblasts was monitored using a ROS detection kit (Beyotime Institute of Biotechnology). Cells in 6-well plates were incubated with variable concentration $\left(10^{-4}, 10^{-6}, 10^{-8} \mathrm{M}\right)$ of DEX for $6 \mathrm{~h}$. Cells were incubated with $10 \mathrm{mM}$ of DCFH-DA at $37^{\circ} \mathrm{C}$ for $20 \mathrm{~min}$, and then washed with PBS. Intracellular ROS level was measured by an inverted fluorescence microscope (IX71; Olympus, Tokyo, Japan). All images were captured under the same conditions, and the fluorescence was quantified by Image J software (National Institutes of Health, Bethesda, MD, USA). Average fluorescence intensity was calculated based on the data from the six fields of each treatment. Three independent experiments were conducted.

Protein extraction and western blot analysis. The treated hFOB 1.19 cells were collected and lysed. The extract was centrifuged $\left(20,000 \mathrm{x} \mathrm{g}\right.$, at $4^{\circ} \mathrm{C}$ for $\left.20 \mathrm{~min}\right)$, and the supernatant was collected to harvest total protein. The protein concentration in the extract was determined using the BCA protein assay (Wuhan Boster Biological Technology, Ltd., Wuhan, China). An equal amount of protein was resolved using SDS-PAGE and electroblotted onto a PVDF membrane (Millipore, Billerica, MA, USA). The membrane was then incubated overnight at $4^{\circ} \mathrm{C}$ with primary antibody against LC3 (1:1,000), beclin $1(1: 1,000)$ and GAPDH $(1: 2,000)$, respectively. Next, the membrane was incubated with goat anti-rabbit secondary antibody conjugated to horseradish peroxidase (1:5,000; ZSGB-BIO, Beijing, China) for $1 \mathrm{~h}$ at room temperature. The protein signal was enhanced using a chemiluminescene system (DNR MF-ChemiBIS 3.2). The band density was quantified using the ImageJ image processing program. 
A

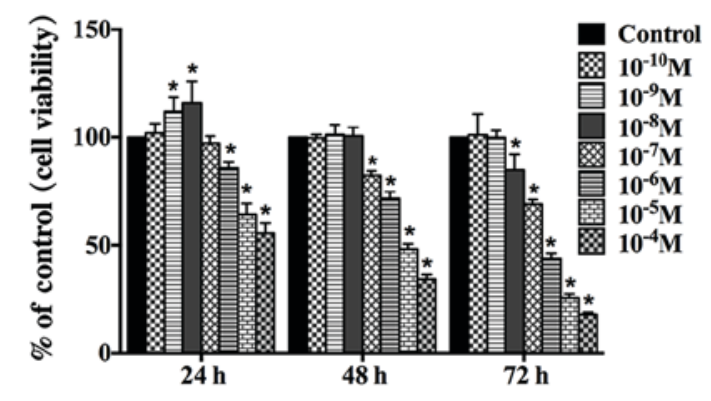

C

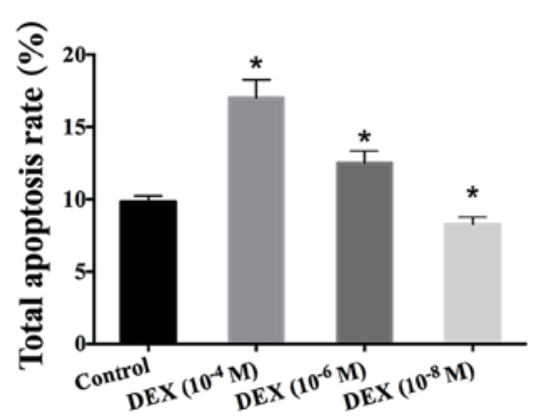

B
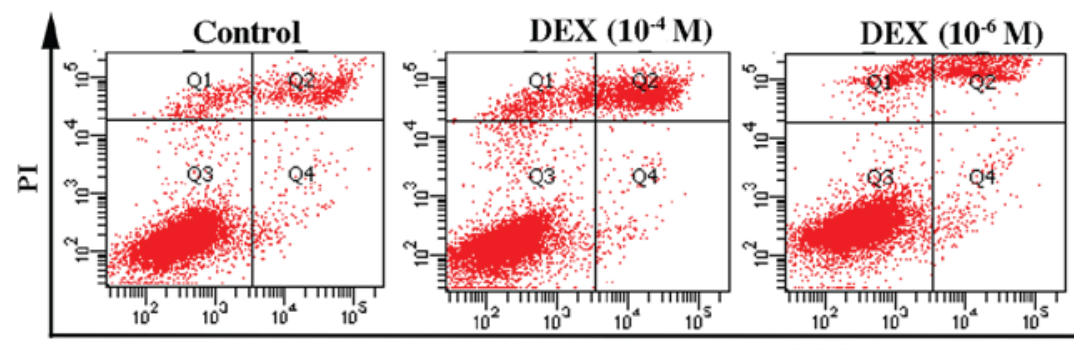

$\operatorname{DEX}\left(1^{-6} \mathrm{M}\right)$

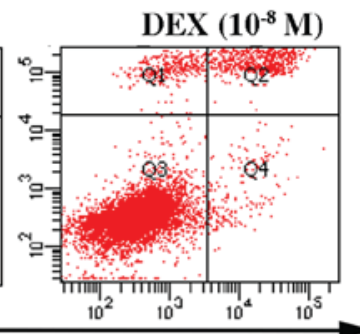

Annexin V-FITC

Figure 1. Effect of DEX on the viability of hFOB 1.19 cells. (A) Cells were treated with various concentration of DEX $\left(10^{-4}-10^{-10} \mathrm{M}\right)$ for different times $(0,24,48$ and $72 \mathrm{~h})$. Cell viability was estimated using an MTT assay. (B) Apoptosis among cells treated with various concentration of DEX $\left(10^{-4}, 10^{-6}\right.$ or $10^{-8} \mathrm{M}$ ) for $24 \mathrm{~h}$ was estimated using Annexin V-FITC/PI staining. (C) Quantification analysis of apoptotic cells. Viable cells, early apoptotic cells, late apoptotic cells and necrotic cells appear in the bottom left quadrant (Q3), bottom right quadrant (Q4), top right quadrant (Q2) and top left quadrant (Q1), respectively. The apoptotic rate was determined as the percentage of Q2 $+\mathrm{Q} 4$. Values are presented as the mean \pm standard deviation from three independent experiments. "P<0.05 vs. control group. FITC, fluorescein isothiocyanate; PI, propidium iodide; DEX, dexamethasone.

Immunofluorescence staining. For immunofluorescence detection, $8 \times 10^{3}$ hFOB 1.19 cells were seeded in a $24-w e l l$ plate and treated with indicated drug for $6 \mathrm{~h}$. The hFOB 1.19 cells were then incubated with anti-LC3 antibody (1:200) at $4^{\circ} \mathrm{C}$ overnight. The secondary antibody, Alexa Fluor ${ }^{\circledR} 488$ Goat Anti-Rabbit IgG (ZSGB-BIO), was applied at room temperature for $1 \mathrm{~h}$, followed by staining the nuclei with DAPI (Beyotime Institute of Biotechnology). Cells were photographed using the inverted fluorescence microscope. LC3-positive cells, defined as cells with visible LC3 foci, were quantified by manually in three randomly-selected fields; nuclei were enumerated by counting DAPI-stained nuclei in the same field. The number of LC3-positive cells in each microscopic field was divided by the number of nuclei in the same field, and was regarded as the rate of LC3-positive cells.

Transmission electron microscopy. Following the incubation with indicated drug for $6 \mathrm{~h}$, the cells were collected and fixed in $5 \%(\mathrm{v} / \mathrm{v})$ glutaraldehyde. Subsequently, the cells were conventionally dehydrated, embedded, sectioned, and stained. The formation of autophagosomes was observed using a transmission electron microscopy (TEM; H-7650; Hitachi, Ltd., Tokyo, Japan). The number of intracellular autophagosomes in every 10 fields was counted.

Statistical analysis. All the experiments were conducted in triple. Quantitative data were presented as mean \pm SD. All statistical analyses were conducted using one-way analysis of variance (ANOVA) by SPSS 24.0 software (IBM, Armonk, NY, USA). Statistical significance was determined using the t-test $(\mathrm{P}<0.05)$.

\section{Results}

Dose-dependent effect of DEX on the viability of hFOB1.19 osteoblasts. To clarify the effect of GCs on osteoblast viability, we used the hFOB1.19 cell line as a cellular model due to these cells' isogeny to human osteoblasts and their strong proliferation ability (27). We treated these cells with different concentrations of DEX $\left(10^{-4}-10^{-8} \mathrm{M}\right)$ for different time $(0$, 24, 48, and $72 \mathrm{~h}$ ) and then assessed cell viability via MTT analysis. Interestingly, our results showed that DEX had a biphasic effect on the viability of hFOB1.19 cells. High-dose DEX $\left(\geq 10^{-6} \mathrm{M}\right)$ continuously and significantly inhibited cell viability. In contrast, the viability of hFOB1.19 cells treated with a low dose of $\operatorname{DEX}\left(10^{-8}, 10^{-9} \mathrm{M}\right)$ was increased at $24 \mathrm{~h}$, and there was no significant difference $(\mathrm{P}>0.05)$ in viability between the $\operatorname{DEX}\left(10^{-8}, 10^{-9} \mathrm{M}\right)$ groups and the control group at $48 \mathrm{~h}$ (Fig. 1A).

To verify that DEX impacts osteoblast viability in a dose-dependent manner, apoptosis of hFOB1.19 cells was estimated via Annexin V-FITC/PI staining after treatment with various concentration of $\operatorname{DEX}\left(10^{-4}, 10^{-6}\right.$, or $\left.10^{-8} \mathrm{M}\right)$ for $24 \mathrm{~h}$. The results indicated that high dose of DEX $\left(\geq 10^{-6} \mathrm{M}\right)$ significantly increased cell apoptosis, which was not significantly elevated in the low-dose $\left(10^{-8} \mathrm{M}\right)$ group compared with that seen in the control group (Fig. 1B and C). Based on these results, we suggest that DEX impacts osteoblast viability in a dose-dependent manner in vitro. High-dose DEX promotes osteoblast apoptosis, whereas the low-dose DEX increases osteoblast viability.

Autophagy is involved in the effect of DEX on osteoblast viability. To investigate whether increased viability of 

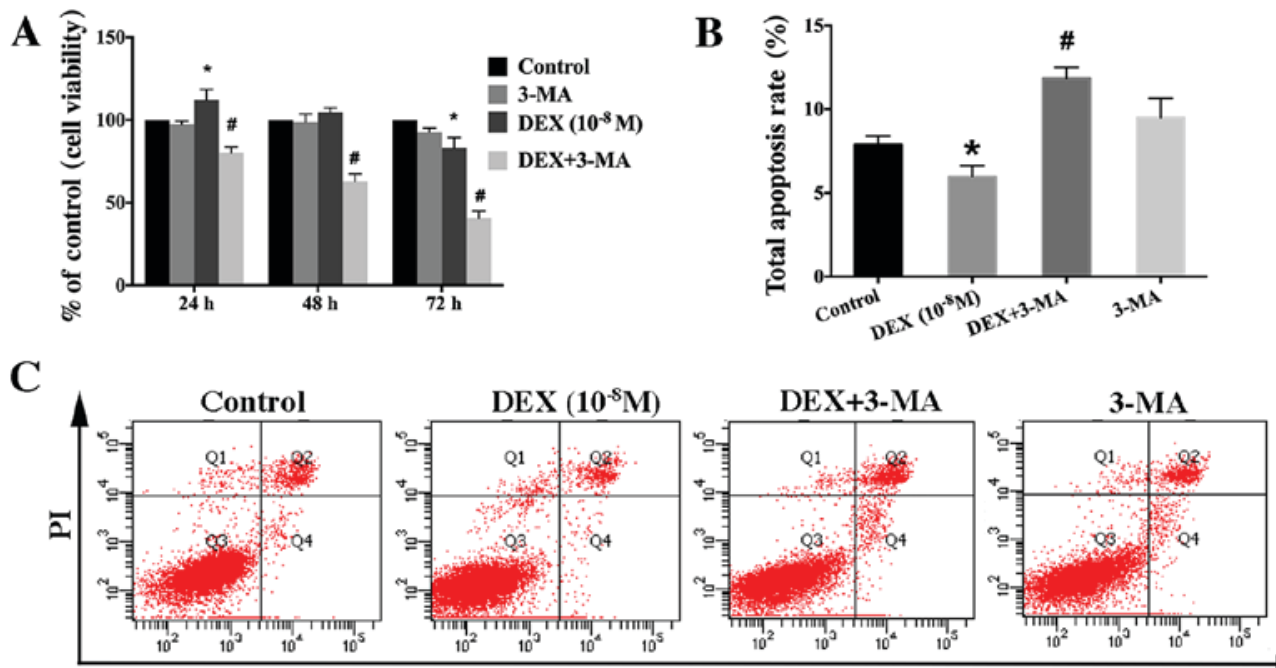

Annexin V-FITC

D
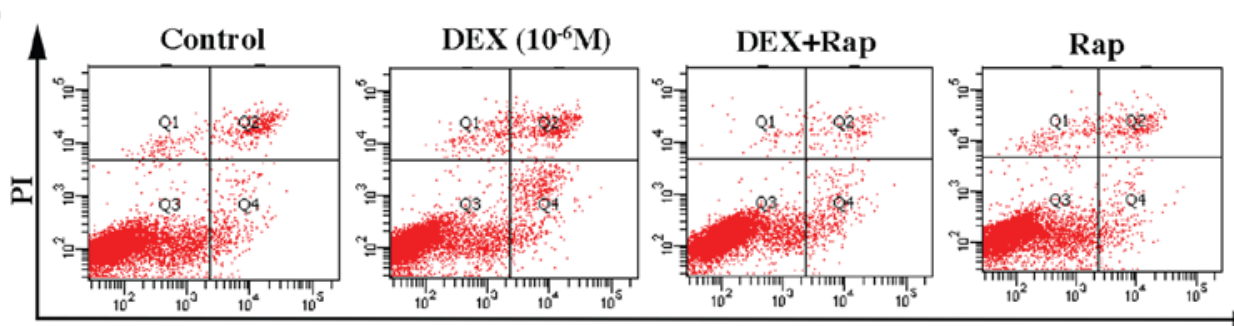

Annexin V-FITC
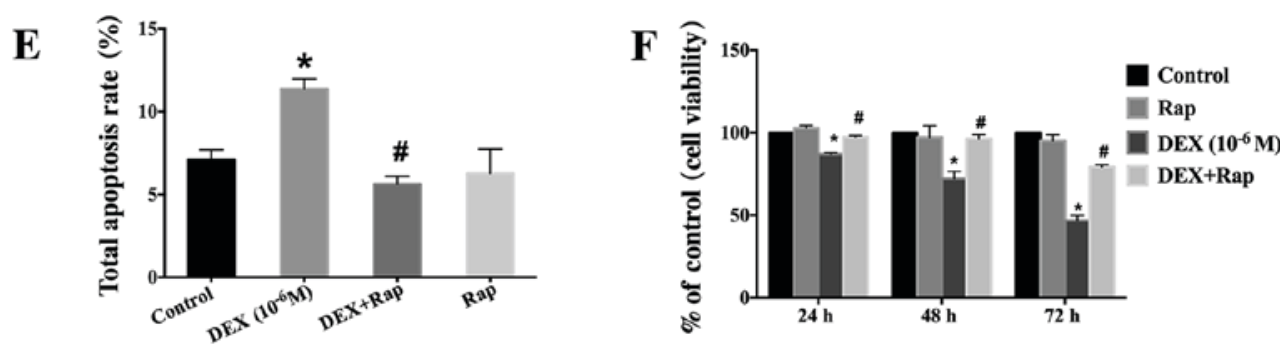

Figure 2. Effect of autophagy modulators on the viability of DEX-treated cells. (A) Cells were treated with DEX $\left(10^{-8} \mathrm{M}\right)$ or DEX $\left(10^{-8} \mathrm{M}+3-\mathrm{MA}(5 \mathrm{mM})\right.$ for 0, 24, 48 or $72 \mathrm{~h}$, and cell viability was estimated using the MTT assay. (B) Quantification analysis of apoptotic cells in 3-MA studies. (C) Apoptosis among cells treated with DEX $\left(10^{-8} \mathrm{M}\right)$ or DEX $\left(10^{-8} \mathrm{M}\right)+3$-MA $(5 \mathrm{mM})$ for $24 \mathrm{~h}$ was estimated using Annexin V-FITC/PI staining. (D) Apoptosis among cells treated with DEX $\left(10^{-6} \mathrm{M}\right)$ or DEX $\left(10^{-6} \mathrm{M}\right)+\operatorname{Rap}(3 \mu \mathrm{M})$ for $24 \mathrm{~h}$ was estimated using Annexin V-FITC/PI staining. (E) Quantification analysis of apoptotic cells in Rap studies. (F) Cells were treated with DEX $\left(10^{-6} \mathrm{M}\right)$ or DEX $\left(10^{-6} \mathrm{M}\right)+$ Rap $(3 \mu \mathrm{M})$ for $0,24,48$ or $72 \mathrm{~h}$, and cell viability was then estimated using the MTT assay. Viable cells, early apoptotic cells, late apoptotic cells and necrotic cells appear in the bottom left quadrant (Q3), bottom right quadrant (Q4), top right quadrant (Q2) and top left quadrant (Q1), respectively. The apoptotic rate was determined as the percentage of Q2 + Q4. Values are presented as the mean \pm standard deviation from three independent experiments. ${ }^{*} \mathrm{P}<0.05$ vs. control group; ${ }^{\text {"}} \mathrm{P}<0.05$ vs. DEX only group. FITC, fluorescein isothiocyanate; PI, propidium iodide; DEX, dexamethasone; Rap, rapamycin; 3-MA, 3-methyladenine.

hFOB1.19 cells induced by a low dose of DEX $\left(10^{-8} \mathrm{M}\right)$ is associated with autophagy, cells were pretreated with 3-MA, an autophagy inhibitor, to inhibit cell autophagy. The results showed that 3-MA significantly inhibited the viability-promoting effect of $\operatorname{DEX}\left(10^{-8} \mathrm{M}\right)$ for hFOB1.19 cells (Fig. 2A). Meanwhile, the results of total apoptotic cells showed that the decrease in apoptotic cells induced by low-dose DEX $\left(10^{-8} \mathrm{M}\right)$ was inhibited by 3 -MA (Fig. $2 \mathrm{~B}$ and C). Therefore, we hypothesize that the dose-dependent effect of DEX on osteoblast viability is closely related to autophagy. To verify this hypothesis, we treated osteoblasts with the specific autophagy agonist Rap. It was observed that the decrease in the viability of hFOB1.19 cells induced by a higher dose of DEX $\left(10^{-6} \mathrm{M}\right)$ was ameliorated (Fig. 2F). In addition, the increased level of apoptotic cells induced by a high dose of DEX $\left(10^{-6} \mathrm{M}\right)$ was alleviated by Rap (Fig. 2D and E). All of these findings demonstrated that autophagy protected hFOB1.19 osteoblasts against DEX by increasing viability and inhibiting apoptosis.

DEX induced autophagy of hFOB1.19 cells in a dose-and time-dependent manner. According to guideline for monitoring autophagy, autophagy level can be accurately evaluated by detecting the autophagic flux, which can be assessed based on protein level of LC3II and beclin 1. To determine whether low dose of DEX $\left(10^{-8} \mathrm{M}\right)$ induce autophagy in hFOB1.19 cells, we examined the protein changes of LC3II and beclin 1 after cells were incubated with $\operatorname{DEX}\left(10^{-8} \mathrm{M}\right)$ for different times $(0$, $1,3,6,12$, and $24 \mathrm{~h}$ ). Western blotting results showed that low dose of DEX $\left(10^{-8} \mathrm{M}\right)$ induced autophagy in a time-dependent manner. The level of LC3II and beclin 1 increased gradually 
A

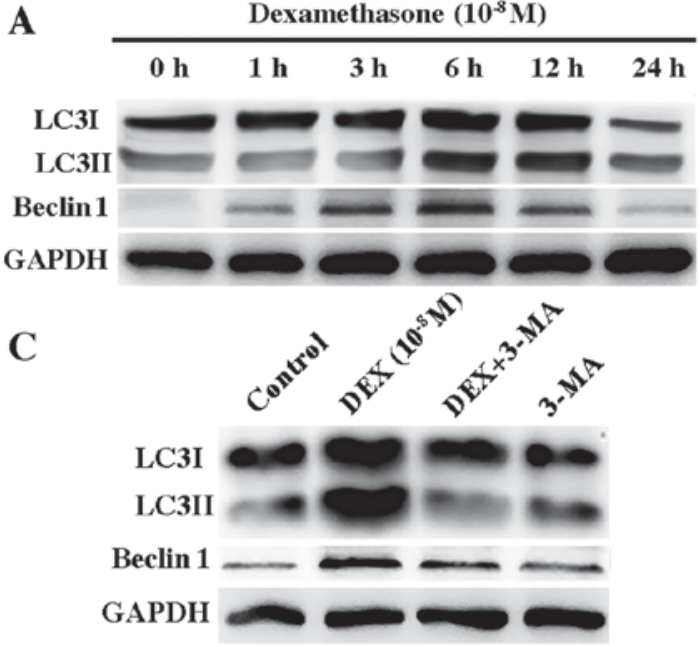

$\mathbf{E}$

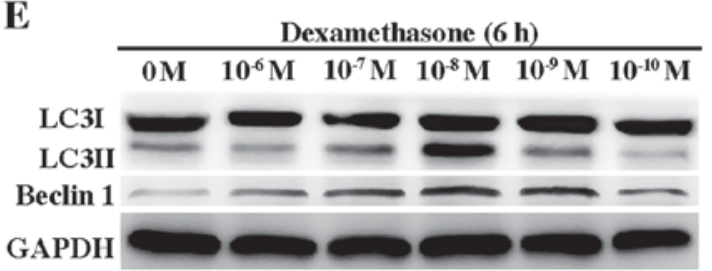

B

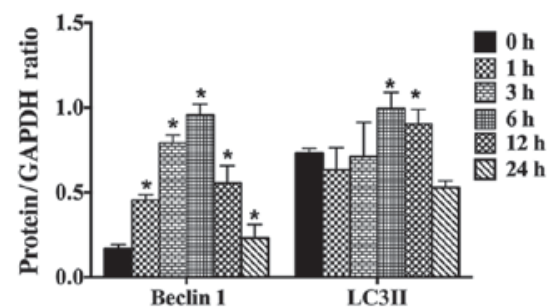

D
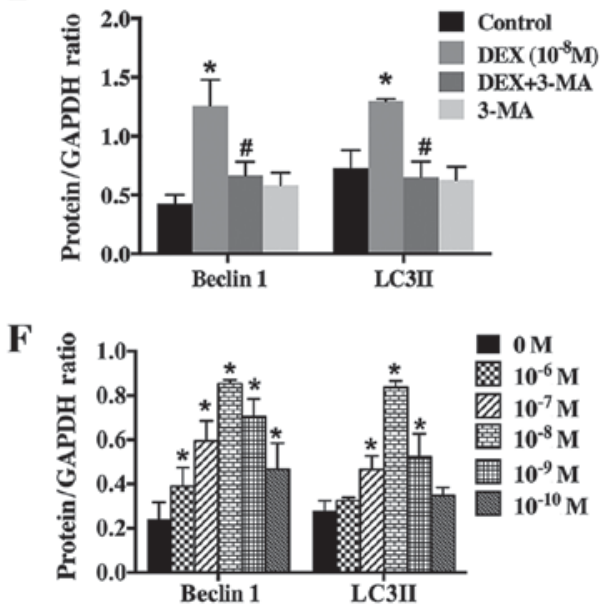

Figure 3. Effect of DEX on the expression of LC3II and beclin 1 in hFOB 1.19 cells. (A) Western blot analysis showing the expression of beclin 1 and LC3 protein in hFOB 1.19 cells treated with DEX $\left(10^{-8} \mathrm{M}\right)$ for $0,1,3,6,12$ or $24 \mathrm{~h}$. (B) Quantitative analysis of beclin 1 and LC3II protein expression. (C) Western blot analysis showing the expression of beclin 1 and LC3 protein in hFOB 1.19 cells treated with $\mathrm{DEX}\left(10^{-8} \mathrm{M}\right)$ or $\mathrm{DEX}\left(10^{-8} \mathrm{M}\right)+3-\mathrm{MA}(5 \mathrm{mM})$ for $6 \mathrm{~h}$. (D) Quantitative analysis of beclin 1 and LC3II protein expression. (E) Western blot analysis showing the expression of beclin 1 and LC3 protein in hFOB 1.19 cells treated with DEX at various concentration $\left(0,10^{-6}, 10^{-7}, 10^{-8}, 10^{-9}\right.$ and $\left.10^{-10} \mathrm{M}\right)$ for 6 h. (F) Quantitative analysis of beclin 1 and LC3II protein expression. Values are presented as the mean \pm standard deviation from three independent experiments. ${ }^{*} \mathrm{P}<0.05$ vs. control; ${ }^{\prime \prime} \mathrm{P}<0.05$ vs. $\mathrm{DEX}\left(10^{-8} \mathrm{M}\right)$ group. DEX, dexamethasone; 3-MA, 3-methyladenine; LC3, light chain 3.

starting from the $3 \mathrm{~h}$ time-point and peaked at the $6 \mathrm{~h}$ timepoint (Fig. 3A and B). However, 3-MA significantly inhibited the autophagy induced by $\operatorname{DEX}\left(10^{-8} \mathrm{M}\right)$ (Fig. $3 \mathrm{C}$ and D).

To further investigate the relationship between DEX and autophagy, we treated hFOB1.19 cells with gradient concentration of DEX $\left(10^{-6}-10^{-10} \mathrm{M}\right)$ for $6 \mathrm{~h}$. Our results showed that autophagy was activated most significantly by $10^{-8} \mathrm{M}$ DEX (Fig. 3E and F). Higher and lower concentration of DEX could also induce autophagy but at lower level, indicating that DEX modulated autophagy in a dose-dependent manner.

The assessment of autolysosome presence and number via transmission electron microscopy (TEM) is recognized as the gold standard for monitoring autophagy intensity. To confirm the role of low dose of DEX $\left(10^{-8} \mathrm{M}\right)$ in inducing autophagy, we used TEM to observe and count autolysosomes. The quantity of autolysosomes was significantly elevated in hFOB1.19 cells treated with DEX $\left(10^{-8} \mathrm{M}\right)$ for $6 \mathrm{~h}$ compared with those in control cells. Furthermore, large numbers of mitochondria that were undergoing phagocytosis were observed. GCs have been reported to increase intracellular oxidative stress by damaging mitochondria (28). In response to this oxidative stress, autophagy is upregulated to help to clear damaged mitochondria with autophagosomes (29). The number of autolysosomes was significantly lower in the group treated with 3-MA (5 mM) than that in the corresponding group treated with DEX alone (Fig. 4A and B). This result confirmed that autophagy protected osteoblasts against oxidative stress by clearing damaged organelles (30). In addition, cells with punctate aggregates (autolysosomes) was defined as LC3-positive cells and they were observed via fluorescence microscopy (Fig. 4C and D). This result was consistent with the finding obtained via TEM.

Autophagy was initiated in response to the increase of ROS induced by low-dose DEX. After hFOB1.19 cells were exposed to various concentration of $\operatorname{DEX}\left(10^{-4}, 10^{-6}\right.$, or $\left.10^{-8} \mathrm{M}\right)$ for $6 \mathrm{~h}$, these cells' intracellular ROS level was recorded by measuring DCF fluorescence intensity. The result showed that intracellular ROS level increased (Fig. 5A and B) as DEX dose increased, suggesting that DEX upregulated the ROS level in a dose-dependent manner.

To further investigate the relationship between ROS and autophagy, we measured ROS level in cells after treatment with Rap or 3-MA. In particular, hFOB1.19 cells were pretreated with Rap (3 $\mu \mathrm{M})$ or 3-MA $(5 \mathrm{mM})$ for $1 \mathrm{~h}$ before incubation with DEX. Rap significantly decreased intracellular ROS level. In contrast, ROS level significantly increased when autophagy was suppressed with 3-MA (Fig. 5C and D). These results indicated that autophagy could protect osteoblasts by decreasing ROS level.

To further investigate the role of ROS in low-dose DEX-induced autophagy, hFOB1.19 cells were pretreated with catalase $(500 \mathrm{U} / \mathrm{ml})$, an ROS inhibitor, for $1 \mathrm{~h}$ prior to treatment with DEX $\left(10^{-8} \mathrm{M}\right)$. Western blot analysis showed that catalase remarkably inhibited the autophagy induced by low-dose DEX (Fig. 6A and B). Furthermore, the result 
A

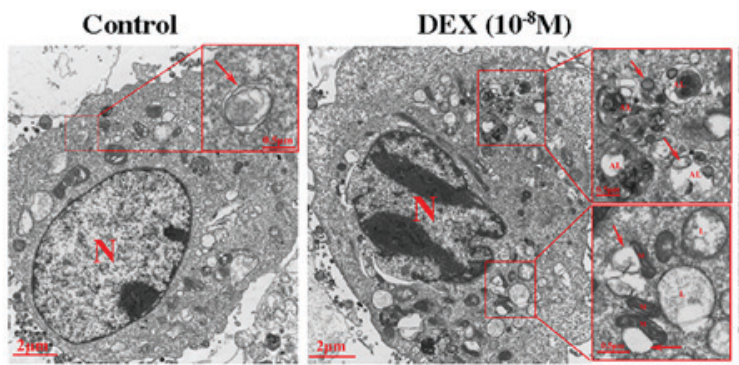

C

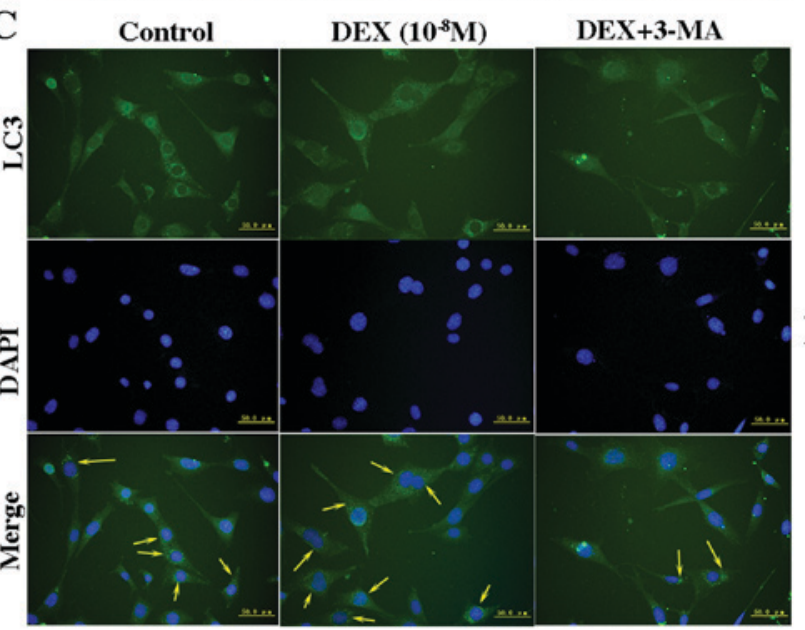

DEX+3-MA

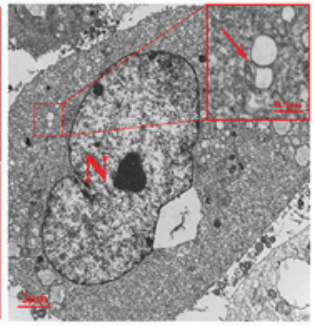

B

Figure 4. Low-dose DEX $\left(10^{-8} \mathrm{M}\right)$-induced autophagy in hFOB 1.19 cells. (A) Transmission electron microscopy showing autophagosomes in hFOB 1.19 cells treated with DEX $\left(10^{-8} \mathrm{M}\right)$ or DEX $\left(10^{-8} \mathrm{M}\right)+3-\mathrm{MA}(5 \mathrm{mM})$ for $6 \mathrm{~h}$. Arrows indicate autophagosomes. Scale bars, main images, $2 \mu \mathrm{m}$; enlarged areas, $0.5 \mu \mathrm{m}$. (B) Quantitation of autophagosomes. (C) Fluorescence microscopy showing LC3-positive cells (cells with visible green fluorescence, LC3 foci) and nuclei (blue fluorescence) in hFOB 1.19 cells treated with DEX $\left(10^{-8} \mathrm{M}\right)$ or DEX $\left(10^{-8} \mathrm{M}\right)+3-\mathrm{MA}(5 \mathrm{mM})$ for $6 \mathrm{~h}$. Arrows indicate LC3-positive cells. Scale bars, $50 \mu \mathrm{m}$. (D) Quantitation of LC3-positive cells. Values are presented as the mean \pm standard from three independent experiments. "P<0.05 vs. control; " $\mathrm{P}<0.05$ vs. DEX only treatment. AL, autophagolysosome; N, nucleus; M, mitochondria; L, lysosome; DEX, dexamethasone; 3-MA, 3-methyladenine; LC3, light chain 3.

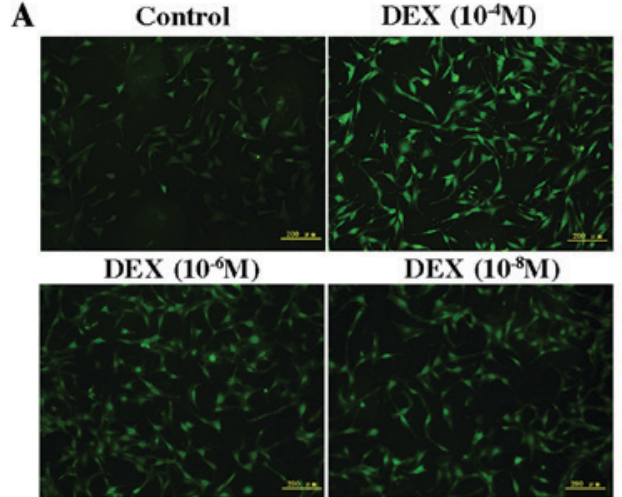

$\mathbf{B}$

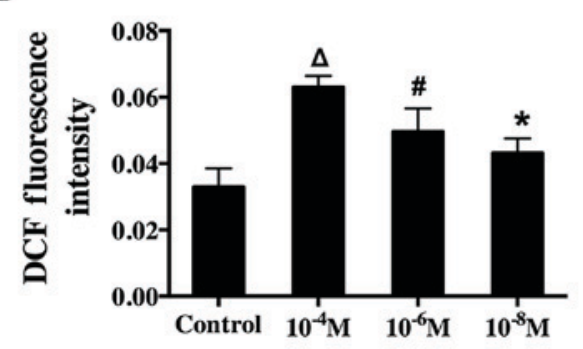

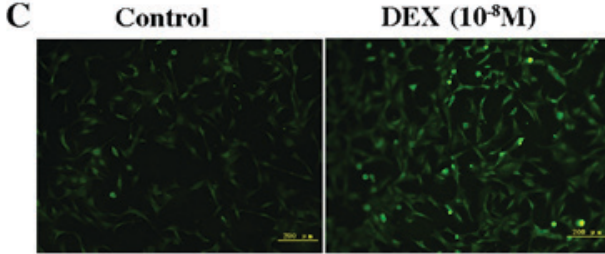

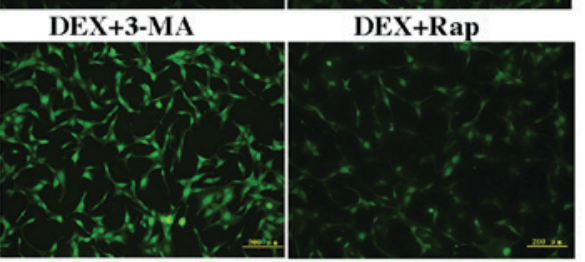

D

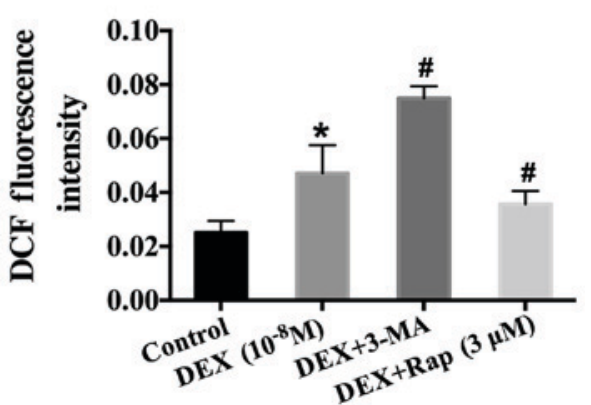

Figure 5. Effect of DEX on intracellular ROS in hFOB 1.19 cells and the effect of the autophagy modulator on the induction of ROS in DEX-treated cells (A) Cells were treated with various concentration of DEX $\left(0,10^{-4}, 10^{-6}\right.$ or $\left.10^{-8} \mathrm{M}\right)$ for $6 \mathrm{~h}$, and ROS levels were determined using 2',7'-dichlorofluorescin diacetate staining. (B) Quantification analysis of ROS. (C) Fluorescence microscopy showing intracellular ROS level in hFOB 1.19 cells treated with DEX $\left(10^{-8} \mathrm{M}\right)$, DEX $\left(10^{-8} \mathrm{M}\right)+3-\mathrm{MA}(5 \mathrm{mM})$ or DEX $\left(10^{-8} \mathrm{M}\right)+\operatorname{Rap}(3 \mu \mathrm{M})$ for $6 \mathrm{~h}$. (D) Quantification analysis of ROS. Scale bars, $200 \mu \mathrm{m}$. Values are presented as the mean \pm standard deviation from three independent experiments. ${ }^{\prime} \mathrm{P}<0.05$ vs. control; ${ }^{*} \mathrm{P}<0.05$ vs. DEX $\left(10^{-8} \mathrm{M}\right)$ treatment; ${ }^{\wedge} \mathrm{P}<0.05$ vs. DEX $\left(10^{-6} \mathrm{M}\right)$ treatment. DEX, dexamethasone; ROS, reactive oxygen species; 3-MA, 3-methyladenine; Rap, rapamycin; DCF, 2',7'-dichlorofluorescin. 
A

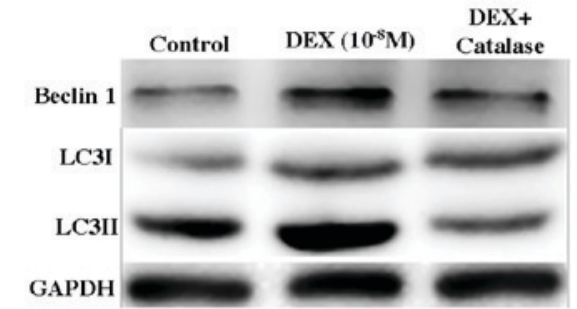

C

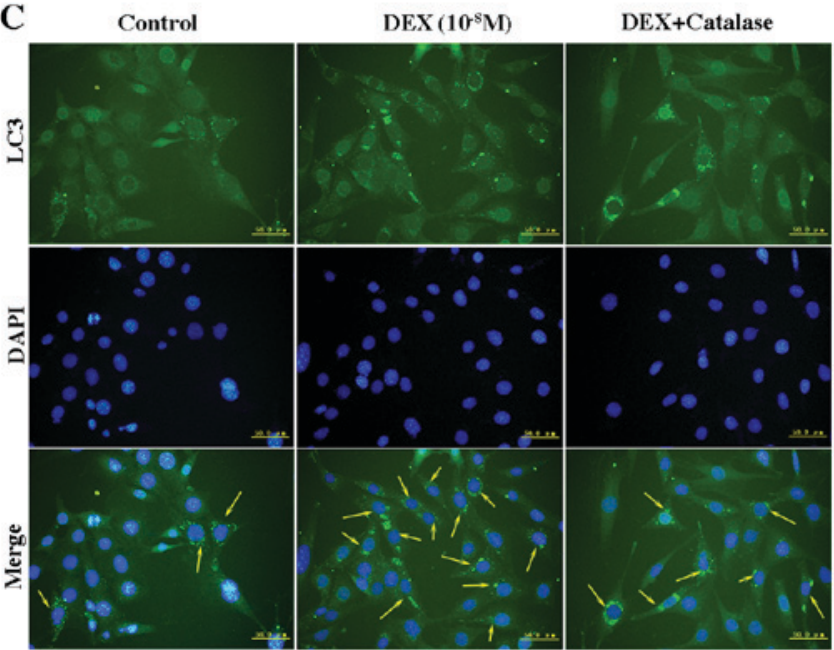

B

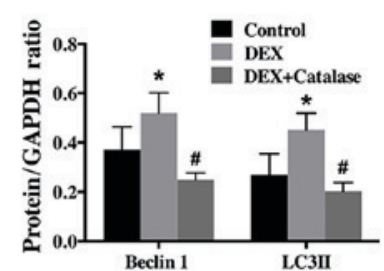

D

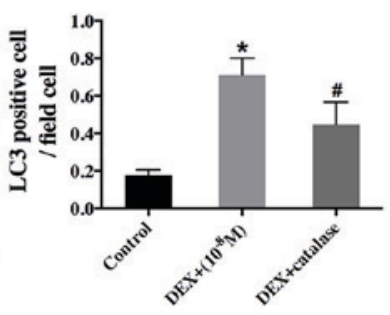

Figure 6. Effect of a ROS inhibitor on autophagy level in low-dose DEX-treated hFOB 1.19 cells. (A) Western blot analysis showing the expression of beclin 1 and LC3 in hFOB 1.19 cells treated with DEX $\left(10^{-8} \mathrm{M}\right)$ or DEX $\left(10^{-8} \mathrm{M}\right)+$ catalase $(500 \mathrm{U} / \mathrm{ml})$ for $6 \mathrm{~h}$. (B) Quantitative analysis of beclin 1 and LC3 protein expression. (C) Fluorescence microscopy showing LC3-positive cells (cells with visible green fluorescence, LC3 foci) and nuclei (blue fluorescence) in hFOB 1.19 cells treated with DEX $\left(10^{-8} \mathrm{M}\right)$ or DEX $\left(10^{-8} \mathrm{M}\right)+$ catalase $(500 \mathrm{U} / \mathrm{ml})$ for $6 \mathrm{~h}$. Arrows indicate LC3-positive cells. Scale bars, $50 \mu \mathrm{m}$. (D) Quantitation of LC3-positive cells. Values are expressed as the mean \pm standard deviation from three independent experiments. "P<0.05 vs. control; ${ }^{\#} \mathrm{P}<0.05$ vs. DEX only treatment. ROS, reactive oxygen species; DEX, dexamethasone; LC3, light chain 3.

\section{(1)}
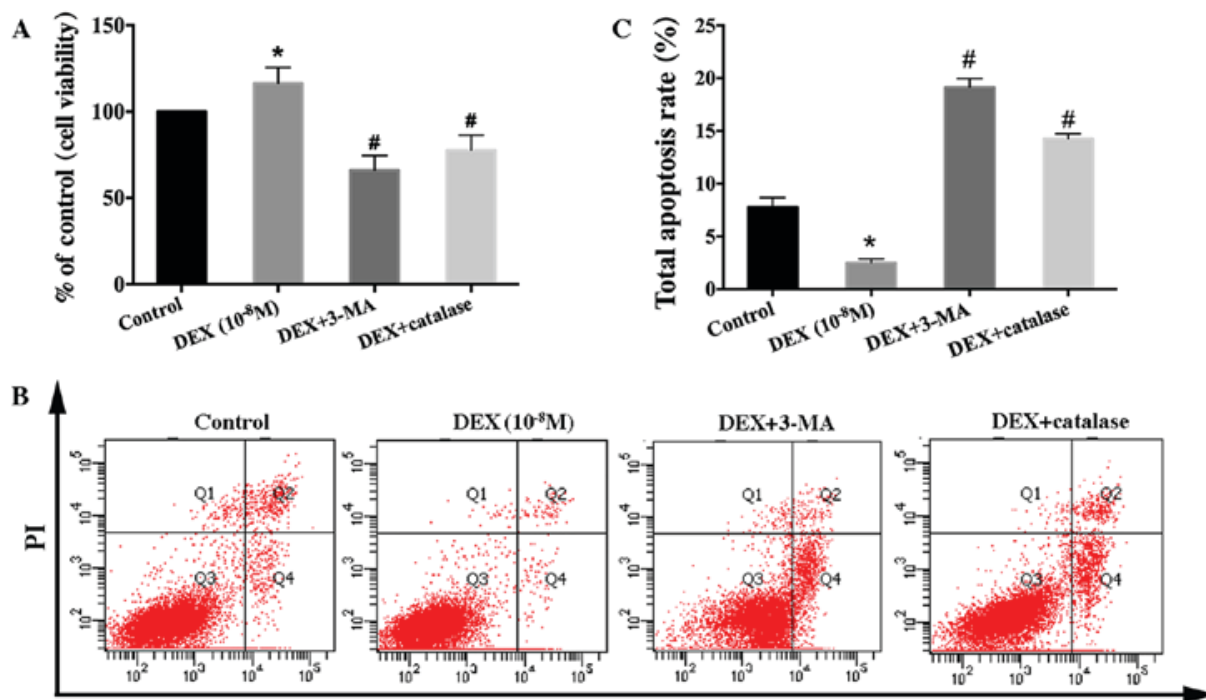

Annexin V-FITC

Figure 7. Effect of a reactive oxygen species inhibitor on the viability and apoptosis of low-dose DEX-treated hFOB 1.19 cells. Cells were treated with DEX $\left(10^{-8} \mathrm{M}\right)$, DEX $\left(10^{-8} \mathrm{M}\right)+3$-MA $(5 \mathrm{mM})$, or DEX $\left(10^{-8} \mathrm{M}\right)+$ catalase $(500 \mathrm{U} / \mathrm{ml})$ for $24 \mathrm{~h}$. (A) Cell viability was estimated using the MTT assay. (B) Cell apoptosis was estimated using Annexin V-FITC/PI staining. (C) Quantification analysis of apoptotic cells. Viable cells, early apoptotic cells, late apoptotic cells and necrotic cells appear in the bottom left quadrant (Q3), bottom right quadrant (Q4), top right quadrant (Q2) and top left quadrant (Q1), respectively. The apoptotic rate was determined as the percentage of $\mathrm{Q} 2+\mathrm{Q} 4$. Values are expressed as the mean \pm standard deviation from three independent experiments. " $\mathrm{P}<0.05$ vs. control group; ${ }^{*} \mathrm{P}<0.05$ vs. DEX $\left(10^{-8} \mathrm{M}\right)$ treatment. FITC, fluorescein isothiocyanate; PI, propidium iodide; DEX, dexamethasone; 3-MA, 3-methyladenine.

of immunofluorescence staining revealed that the rate of LC3-positive cells was lower in the group treated with catalase and DEX than that treated with DEX alone (Fig. 6C and D).
Taken together, the aforementioned results suggested that a low dose of DEX induced autophagy by upregulating intracellular ROS level. 
ROS inhibitor suppressed the increased viability of $h F O B 1.19$ cells induced by low-dose DEX. Based on the previous results, we concluded that low-dose DEX increased hFOB 1.19 cells viability by inducing autophagy via intracellular ROS. To verify the key role of ROS in the improvement of osteoblast viability induced by a low dose of DEX, we firstly investigated cell viability via MTT analysis. Significantly lower viability was observed in hFOB1.19 cells co-treated with catalase (500 U/ml) and DEX (10-8 M), which was consistent with 3-MA group (Fig. 7A). The same result was obtained in Annexin V-FITC/PI staining analyses; in particular, more apoptotic cells were observed after treatment with catalase and DEX than treatment with DEX alone (Fig. 7B and C). All of our results confirmed that low-dose DEX upregulated the viability of hFOB1.19 cells by increasing intracellular ROS.

\section{Discussion}

Many studies have suggested that GCs contributed to the development and progression of GIOP by repressing viability and promoting apoptosis among osteoblasts $(2,6)$. However, there is controversy regarding the effect of GCs on osteoblasts in vivo and in vitro. Endogenous GCs, which are secreted by the adrenal cortex, are essential for bone development $(31,32)$. Moreover, investigations have found that in vitro, DEX can accelerate the osteogenic activity of osteoblasts (33) and the proliferation of bone marrow stromal cells (34), which are precursors and source of osteoblasts. Our results showed that DEX exerted a biphasic effect on the viability of hFOB1.19 cells: High-dose DEX $\left(\geq 10^{-6} \mathrm{M}\right)$ continuously inhibited cell viability and markedly accelerated apoptosis, whereas lower-dose DEX $\left(10^{-8} \mathrm{M}\right)$ increased cell viability in the early stage, implying that both dose and treatment time contributed to the effect of GCs on osteoblast viability. This result is similar to the finding obtained by Jia et al (8) and Shi et al (9), who claimed that the effect of GCs on cell viability was determined by GCs dose. In fact, the treatment dose of GCs used for autoimmune and inflammatory diseases is higher than the physiological level of these compounds, and treatment duration is often months or even years. This observation may partially resolve the controversy regarding the opposite effect of GCs on osteoblast viability.

As we know, basal autophagy occurs at low level in all cells and is considered as a quality control mechanism of protein and organelle, maintaining normal cellular homeostasis (12). When encountering some stresses, autophagy is usually upregulated (35). If autophagy is excessively upregulated, autophagosomes will devour the cellular proteins and organelles, and cause cells apoptosis. Hence, autophagy is also regarded as type II form of programmed cell death (36). However, under some moderate stimulus conditions, autophagy can be moderately induced to promote cell survival and avert apoptosis by eliminating stress inducers (37). Autophagy ensures the delivery of metabolic substrates to cells in order to fulfill their energy demand during stress, thus supports cell growth and survival $(38,39)$. This function is critical for the survival of some terminally differentiated cells, such as osteoblasts and osteoclasts (40). Many recent studies have demonstrated the protective effect of autophagy with respect to facilitating osteoblast viability under various stress $(16,17,41)$. Moreover, the involvement of GC-induced autophagy in modulating the viability of various cells has been well demonstrated (42-44).

However, the role of autophagy in affecting the viability of osteoblasts exposed to low dose of GCs has rarely been reported. Based on prior studies and our results, we hypothesize that the increased viability of osteoblasts exposed to low-dose DEX may be associated with autophagy. Our results indicated that treatment with 3-MA markedly inhibited the increased osteoblast activity induced by a low dose of $\operatorname{DEX}\left(10^{-8} \mathrm{M}\right)$ and significantly increased the apoptosis rate; both effects verified our hypothesis. In conclusion, all of the aforementioned results indicated that a low dose of GCs increased osteoblast viability by inducing autophagy at early stage.

DEX, as an inducer of oxidative stress, can lead to mitochondrial dysfunction and upregulate oxidative stress in cells $(21,45)$. Therefore, it could be observed that intracellular ROS level was consistently upregulated as DEX dose increased. To respond to this oxidative stress, autophagy was upregulated to protect osteoblasts from apoptosis due to DEX exposure; this phenomenon could be observed by detecting autophagy-related proteins and autophagosomes in osteoblasts incubated with low-dose DEX. If the DEX dose and the corresponding oxidative stress are low and the treatment time is short, this attempt to protect osteoblasts will be successful. However, as DEX dose and treatment time increase, the antioxidant capacity of autophagy will be overwhelmed, and the ability of protecting against stress will be reduced, potentially leading to osteoblast apoptosis. Our study demonstrated that osteoblast viability was strictly determined by GCs dose. A low dose of GCs activated autophagy to protect cells from apoptosis, whereas a high dose of GCs induced apoptosis. This finding was consistent with the results of an in vivo investigation by Jia et al (8), who reported that autophagic gene expression and osteocyte autophagy increased when mice were treated with a low dose of GCs but the expression of apoptotic genes increased when mice were treated with higher dose of GCs.

The generation of ROS is known to be a normal physiological activity of cellular metabolism (46). However, researches on the pathological mechanism of GCs have demonstrated that excessive and long-term treatment with GCs caused the excessive accumulation of intracellular ROS by dysregulating mitochondria function, leading to the upregulation of oxidative stress (21). Similarly, our results showed that ROS level was increased in hFOB1.19 cells treated with DEX. Moreover, the higher DEX dose, the higher ROS level. The excessive accumulation of ROS might disturb cellular homeostasis, resulting in cell injury and death $(47,48)$. Both in vitro and in vivo studies have demonstrated that excessive ROS and oxidative stress reduce bone formation by inhibiting the differentiation and viability of osteoblasts $(45,49)$. Therefore, we concluded that the reduced viability of osteoblasts treated with high-dose DEX was associated with excessive intracellular ROS.

However, studies have showed that physiological ROS acted as intracellular signalling molecule, and moderate ROS was thought to modulate cell viability by upregulating autophagy $(17,46,50)$. Based on our investigation and works by other researchers, we hypothesize that low-dose DEX increased osteoblast activity by inducing autophagy via intracellular ROS. 
To verify this hypothesis, we treated osteoblasts with a low dose of DEX $\left(10^{-8} \mathrm{M}\right)$ combined with or without the ROS scavenger catalase. The results showed that the increased cell activity and autophagy induced by a low dose of DEX was significantly inhibited by catalase. In addition, increased autophagy can decrease or eliminate intracellular ROS (51). In our study, the upregulation of autophagy by Rap caused the reduction of intracellular ROS in osteoblasts treated with low-dose DEX, whereas pretreatment with 3-MA produced the opposite result. In summary, our data implied that the low-dose GCs induced autophagy via intracellular ROS and autophagy protected osteoblasts from apoptosis by reducing ROS level.

In conclusion, the present study demonstrated that GCs modulated osteoblast viability in a dose-dependent manner. High-dose of GCs induced osteoblast apoptosis, whereas a lower dose of GCs enhanced osteoblast viability by inducing autophagy via intracellular ROS. Our findings suggested that the modulation of osteoblast autophagy could be a novel approach for the prevention and treatment of GIOP. Next, further studies should be performed to explore the underlying mechanism of low-dose GC-induced autophagy via ROS.

\section{References}

1. Kenanidis E, Potoupnis ME, Kakoulidis P, Leonidou A, Sakellariou GT, Sayegh FE and Tsiridis E: Management of glucocorticoid-induced osteoporosis: Clinical data in relation to disease demographics, bone mineral density and fracture risk. Expert Opin Drug Saf 14: 1035-1053, 2015.

2. Frenkel B, White W and Tuckermann J: Glucocorticoid-induced osteoporosis. Adv Exp Med Biol 872: 179-215, 2015.

3. Dalle Carbonare L, Arlot ME, Chavassieux PM, Roux JP, Portero NR and Meunier PJ: Comparison of trabecular bone microarchitecture and remodeling in glucocorticoid-induced and postmenopausal osteoporosis. J Bone Miner Res 16: 97-103, 2001.

4. Van Staa TP, Laan RF, Barton IP, Cohen S, Reid DM and Cooper C: Bone density threshold and other predictors of vertebral fracture in patients receiving oral glucocorticoid therapy. Arthritis Rheum 48: 3224-3229, 2003.

5. Capulli M, Paone R and Rucci N: Osteoblast and osteocyte: Games without frontiers. Arch Biochem Biophys 561: 3-12, 2014.

6. Moriishi T and Komori T: Glucocorticoid and bone. The inhibition of osteoblast differentiation and induction of osteocyte apoptosis through the regulation of $\mathrm{Bcl}-2$ by glucocorticoids Clin Calcium 24: 1329-1336, 2014 (In Japanese).

7. Komori T: Glucocorticoid signaling and bone biology. Horm Metab Res 48: 755-763, 2016.

8. Jia J, Yao W, Guan M, Dai W, Shahnazari M, Kar R, Bonewald L, Jiang JX and Lane NE: Glucocorticoid dose determines osteocyte cell fate. FASEB J 25: 3366-3376, 2011.

9. Shi J, Wang L, Zhang H, Jie Q, Li X, Shi Q, Huang Q, Gao B, Han Y, Guo K, et al: Glucocorticoids: Dose-related effects on osteoclast formation and function via reactive oxygen species and autophagy. Bone 79: 222-232, 2015.

10. Martinez-Lopez N, Athonvarangkul D and Singh R: Autophagy and aging. Adv Exp Med Biol 847: 73-87, 2015.

11. Wang JY, Yao WX, Wang Y, Fan YL and Wu JB: Network analysis reveals crosstalk between autophagy genes and disease genes. Sci Rep 7: 44391, 2017.

12. Lee JY, Koga H, Kawaguchi Y, Tang W, Wong E, Gao YS, Pandey UB, Kaushik S, Tresse E, Lu J, et al: HDAC6 controls autophagosome maturation essential for ubiquitin-selective quality-control autophagy. EMBO J 29: 969-980, 2010.

13. Degenhardt K, Mathew R, Beaudoin B, Bray K, Anderson D, Chen G, Mukherjee C, Shi Y, Gélinas C, Fan Y, et al: Autophagy promotes tumor cell survival and restricts necrosis, inflammation, and tumorigenesis. Cancer Cell 10: 51-64, 2006.

14. Sid B, Verrax J and Calderon PB: Role of AMPK activation in oxidative cell damage: Implications for alcohol-induced liver disease. Biochem Pharmacol 86: 200-209, 2013.
15. Kouroku Y, Fujita E, Tanida I, Ueno T, Isoai A, Kumagai H, Ogawa S, Kaufman RJ, Kominami E and Momoi T: ER stress (PERK/eIF2alpha phosphorylation) mediates the polyglutamine-induced LC3 conversion, an essential step for autophagy formation. Cell Death Differ 14: 230-239, 2007.

16. Gu X, Han D, Chen W, Zhang L, Lin Q, Gao J, Fanning S and Han B: SIRT1-mediated FoxOs pathways protect against apoptosis by promoting autophagy in osteoblast-like MC3T3-E1 cells exposed to sodium fluoride. Oncotarget 7: 65218-65230, 2016.

17. Yang L, Meng $\mathrm{H}$ and Yang M: Autophagy protects osteoblasts from advanced glycation end products-induced apoptosis through intracellular reactive oxygen species. J Mol Endocrinol 56: 291-300, 2016

18. Klionsky DJ, Abdelmohsen K, Abe A, Abedin MJ, Abeliovich $\mathrm{H}$, Acevedo Arozena A, Adachi H, Adams CM, Adams PD, Adeli K, et al: Guidelines for the use and interpretation of assays for monitoring autophagy (3rd edition). Autophagy 12: 1-222, 2016.

19. Tanida I, Ueno T and Kominami E: LC3 and autophagy. Methods Mol Biol 445: 77-88, 2008.

20. Fu LL, Cheng Y and Liu B: Beclin-1: Autophagic regulator and therapeutic target in cancer. Int J Biochem Cell Biol 45: 921-924, 2013.

21. Grishko V, Xu M, Ho R, Mates A, Watson S, Kim JT, Wilson GL and Pearsall AW IV: Effects of hyaluronic acid on mitochondrial function and mitochondria-driven apoptosis following oxidative stress in human chondrocytes. J Biol Chem 284: 9132-9139, 2009.

22. Ong SL, Zhang Y and Whitworth JA: Reactive oxygen species and glucocorticoid-induced hypertension. Clin Exp Pharmacol Physiol 35: 477-482, 2008.

23. Henrotin Y, Kurz B and Aigner T: Oxygen and reactive oxygen species in cartilage degradation: Friends or foes? Osteoarthritis Cartilage 13: 643-654, 2005.

24. Ghavami S, Shojaei S, Yeganeh B, Ande SR, Jangamreddy JR, Mehrpour M, Christoffersson J, Chaabane W, Moghadam AR, Kashani HH, et al: Autophagy and apoptosis dysfunction in neurodegenerative disorders. Prog Neurobiol 112: 24-49, 2014.

25. Almeida M, Han L, Martin-Millan M, Plotkin LI, Stewart SA, Roberson PK, Kousteni S, O'Brien CA, Bellido T, Parfitt AM, et al: Skeletal involution by age-associated oxidative stress and its acceleration by loss of sex steroids. J Biol Chem 282: 27285-27297, 2007.

26. Wauquier F, Leotoing L, Coxam V, Guicheux J and Wittrant Y: Oxidative stress in bone remodelling and disease. Trends Mol Med 15: 468-477, 2009.

27. Harris SA, Enger RJ, Riggs BL and Spelsberg TC: Development and characterization of a conditionally immortalized human fetal osteoblastic cell line. J Bone Miner Res 10: 178-186, 1995.

28. Brand MD, Affourtit C, Esteves TC, Green K, Lambert AJ, Miwa S, Pakay JL and Parker N: Mitochondrial superoxide: Production, biological effects, and activation of uncoupling proteins. Free Radic Biol Med 37: 755-767, 2004.

29. Wohlgemuth SE, Calvani $R$ and Marzetti E: The interplay between autophagy and mitochondrial dysfunction in oxidative stress-induced cardiac aging and pathology. J Mol Cell Cardiol 71: 62-70, 2014

30. Mizushima N, Levine B, Cuervo AM and Klionsky DJ: Autophagy fights disease through cellular self-digestion. Nature 451: 1069-1075, 2008

31. Kalak R, Zhou H, Street J, Day RE, Modzelewski JR, Spies CM, Liu PY, Li G, Dunstan CR and Seibel MJ: Endogenous glucocorticoid signalling in osteoblasts is necessary to maintain normal bone structure in mice. Bone 45: 61-67, 2009.

32. Zhou H, Mak W, Kalak R, Street J, Fong-Yee C, Zheng Y, Dunstan CR and Seibel MJ: Glucocorticoid-dependent Wnt signaling by mature osteoblasts is a key regulator of cranial skeletal development in mice. Development 136: 427-436, 2009.

33. Ishida Y, Tertinegg I and Heersche JN: Progesterone and dexamethasone stimulate proliferation and differentiation of osteoprogenitors and progenitors for adipocytes and macrophages in cell populations derived from adult rat vertebrae. J Bone Miner Res 11: 921-930, 1996.

34. Atmani H, Chappard D and Basle MF: Proliferation and differentiation of osteoblasts and adipocytes in rat bone marrow stromal cell cultures: Effects of dexamethasone and calcitriol. J Cell Biochem 89: 364-372, 2003.

35. Wang F, Jia J and Rodrigues B: Autophagy, metabolic disease, and pathogenesis of heart dysfunction. Can J Cardiol 33: 850-859, 2017. 
36. Booth LA, Tavallai S, Hamed HA, Cruickshanks N and Dent P. The role of cell signalling in the crosstalk between autophagy and apoptosis. Cell Signal 26: 549-555, 2014

37. Bhutia SK, Kegelman TP, Das SK, Azab B, Su ZZ, Lee SG, Sarkar D and Fisher PB: Astrocyte elevated gene-1 induces protective autophagy. Proc Natl Acad Sci USA 107: 22243-22248, 2010.

38. Ouyang L, Shi Z, Zhao S, Wang FT, Zhou TT, Liu B and Bao JK: Programmed cell death pathways in cancer: A review of apoptosis, autophagy and programmed necrosis. Cell Prolif 45: 487-498, 2012.

39. Bhutia SK, Mukhopadhyay S, Sinha N, Das DN, Panda PK Patra SK, Maiti TK, Mandal M, Dent P, Wang XY, et al: Autophagy: Cancer's friend or foe? Adv Cancer Res 118: 61-95, 2013.

40. Manolagas SC and Parfitt AM: What old means to bone. Trends Endocrinol Metab 21: 369-374, 2010.

41. Lv XH, Zhao DH, Cai SZ, Luo SY, You T, Xu BL and Chen K: Autophagy plays a protective role in cell death of osteoblasts exposure to lead chloride. Toxicol Lett 239: 131-140, 2015.

42. Wang L, Fan J, Lin YS, Guo YS, Gao B, Shi QY, Wei BY, Chen L, Yang L, Liu J and Luo ZJ: Glucocorticoids induce autophagy in rat bone marrow mesenchymal stem cells. Mol Med Rep 11: 2711-2716, 2015.

43. Zhao Y, Zuo Y, Huo HJ, Xiao YL, Yang XJ and Xin DQ: Glucocorticoid induced autophagy in N1511 chondrocyte cells. Eur Rev Med Pharmacol Sci 18: 3573-3579, 2014.

44. Grander D, Kharaziha P, Laane E, Pokrovskaja K and Panaretakis T: Autophagy as the main means of cytotoxicity by glucocorticoids in hematological malignancies. Autophagy 5: 1198-1200, 2009.
45. Almeida M, Han L, Ambrogini E, Weinstein RS and Manolagas SC: Glucocorticoids and tumor necrosis factor alpha increase oxidative stress and suppress Wnt protein signaling in osteoblasts. J Biol Chem 286: 44326-44335, 2011.

46. Kiffin R, Bandyopadhyay U and Cuervo AM: Oxidative stress and autophagy. Antioxid Redox Signal 8: 152-162, 2006.

47. Zhen YF, Wang GD, Zhu LQ, Tan SP, Zhang FY, Zhou XZ and Wang XD: P53 dependent mitochondrial permeability transition pore opening is required for dexamethasone-induced death of osteoblasts. J Cell Physiol 229: 1475-1483, 2014.

48. Manolagas SC: From estrogen-centric to aging and oxidative stress: A revised perspective of the pathogenesis of osteoporosis. Endocr Rev 31: 266-300, 2010.

49. Schroder K: NADPH oxidases in bone homeostasis and osteoporosis. Cell Mol Life Sci 72: 25-38, 2015.

50. Suzuki M, Bandoski C and Bartlett JD: Fluoride induces oxidative damage and SIRT1/autophagy through ROS-mediated JNK signaling. Free Radic Biol Med 89: 369-378, 2015.

51. Scherz-Shouval R and Elazar Z: ROS, mitochondria and the regulation of autophagy. Trends Cell Biol 17: 422-427, 2007.

This work is licensed under a Creative Commons Attribution-NonCommercial-NoDerivatives 4.0 International (CC BY-NC-ND 4.0) License. 